und unregelmäßig erscheinende Zielreize (kleiner bzw. großer blauer Kreis) sowie alkoholische (Bier, Wein, Likör) und nichtalkoholische (Wasser, Kaffee, Soda) Ablenkungsreize gezeigt. Die Probanden wurden aufgefordert, bei allen unregelmäßig erscheinenden Reizen einen Knopf zu drücken. Die Alkoholabhängigkeit wurde mittels des Michigan Alcohol Screening Tests, der Alcohol Dependence Scale und der Alcohol Craving Scale in Stadien eingeteilt.

Kontrollen und Langzeitabstinente zeigten im Vergleich zu aktuellen Trinkern bei Präsentation von alkoholischen Distraktoren eine signifikant erhöhte Aktivierung von Hirnregionen, die Aufmerksamkeits- und kognitiven Kontrolle dienen (inferiorer Parietallappen und dorsales ACC), während aktuell Abstinente und Langzeitabstinente eine verminderte Rekrutierung von limbischen Regionen (Amygdala), die für die affektive Verarbeitung von Alkoholreizen zu- ständig sind, zeigten. Dies bedeutet, dass mit zunehmender Abstinenzdauer eine erhöhte kognitive Kontrolle und eine stärkere Regulation von limbischen Antworten auf Alkoholreize eintreten. Bei aktuellen Trinkern hingegen zeigt sich die kognitive Kontrolle und parietale Aufmerksamkeit vermindert, was eine erhöhte Vulnerabilität dieser Gruppe für die Präsentation von Alkoholschlüsselreizen darstellt. In allen Phasen der Abhängigkeit korrelierte das Ausmaß des Cravings positiv mit den reizbezogenen Aktivierungen im ventralen ACC, dem medialen präfrontalen Cortex und dem Zerebellum.

Kommentar: In dieser Studie wurden erstmalig Alkoholschlüsselreize als aufgabenunabhängige Zufallsreize in einer Aufmerksamkeitsaufgabe präsentiert und die darauf ansprechenden Hirnregionen identifiziert. Im Detail konnte gezeigt werden, dass die zerebrale Verarbeitung solcher Reize von der aktuellen Phase der Alkoholabhängigkeit abhängt. Die Studie liefert einen interessanten Beitrag zu den zugrunde liegenden neuronalen Mechanismen der Genesung von Alkoholabhängigkeit.

Die Einschränkung der Studie besteht vor allem in den kleinen Gruppengrößen, sodass andere Zusammenhänge mit kleineren Effektstärken möglicherweise nicht aufgedeckt werden konnten und die Generalisierung der Ergebnisse auf ein großes Kollektiv von alkoholabhängigen Patienten eingeschränkt zu werten ist. Weiterhin kann das Querschnittsdesign der Studie nicht zwischen den Hirnaktivierungen der Regenerationsphase und der prämorbiden Phasen der Alkoholabhängigkeit differenzieren. Hierfür würde man ein longitudinales Studiendesign benötigen. Dr. med. Christina Stößel

Fryer SL, et al. Differential brain response to alcohol cue distractors across stages of alcohol dependence. Biol Psychol. 2012; 92 (2): 282-91

\title{
Money, money, money! Hohe Preise - weniger Konsum
}

\author{
Am Beispiel Kanada wird gezeigt, wie sich staatlich festgelegte Preispolitik auf das \\ Konsummuster von Alkohol auswirkt. Die Studie gibt damit einen interessanten Denkanstoß \\ zur möglichen Verbesserung der Gesundheit in der Allgemeinbevölkerung.
}

$\mathrm{E}_{2}^{\mathrm{in}}$ ine kanadische Forschergruppe hat zum ersten Mal konkret den Zusammenhang zwischen Preis und Alkoholkonsum pro Kopf erforscht. Ihre Ausgangsthese: Die Erhöhung einer Preisuntergrenze für alkoholische Getränke dämmt den Alkoholkonsum ein. In British Columbia/Kanada besteht eine strenge Alkoholverkaufskontrolle mit staatlich festgesetzten Preisuntergrenzen, die in den letzten 20 Jahren eine intermittierende Anpassung erfahren haben. Alkohol kann nur in den 200 staatlichen Läden der BC Liquor Distribution Branch (BC LDB) gekauft werden, die auch die Einhaltung der Preisuntergrenzen in den über 600 privaten Getränkeläden überwacht. Die Autoren errechneten nun für den Zeitraum 1989 bis 2010 aus den Verkaufszahlen der BC LDB, den Preisuntergrenzen und der Gesamtbevölkerung (über 15-Jährige) den Pro-Kopf-Verbrauch an Alkohol und bestimmten den Einfluss der Preiserhöhungen auf das Konsummuster.

Ein Preisanstieg von $10 \%$ bei einem alkoholischen Getränk reduzierte dessen Konsum um 16,1 \% (p<0.001). Unter der Annahme, dass nach einem Preisanstieg auf andere Arten von Alkohol ausgewichen wird, untersuchten die Autoren den Effekt einer generellen Preiserhöhung um $10 \%$ für alle Arten von alkoholischen Getränken. Es zeigte sich eine Reduktion im Konsum von zum Beispiel Wein um $8,9 \%(p=0,033)$, Schnaps um 6,8\% $(p=0,004)$ und Bier um 1,5\% $(p=0,043)$. Die Autoren errechneten insgesamt eine Verringerung des Alkoholkonsums der Allgemeinbevölkerung um 3,4\% als Folge der $10 \%$ igen Preissteigerung.

Kommentar: Die vorgestellte Studie ist die erste, die anhand einer breiten Datenlage und eines langen Zeitraumes die Beziehung zwischen Preisgestaltung und Alkoholkonsum in konkreten Zahlen darstellen kann.
Gerade der lange Beobachtungszeitraum stellt eine wesentliche Stärke der Studie dar. Als Einschränkung kann gesehen werden, dass der Alkoholpreis nicht auf eine Standardwarengröße, also auf die allgemeinen Haushalts- oder Lebenshaltungskosten bezogen wurde. Einen Hinweis auf die konkreten Auswirkungen im Gesundheitswesen, wie eine mögliche Reduktion von Abhängigkeitserkrankungen, gibt die Studie zwar nicht, unter der Erkenntnis, dass besonders Alkoholabhängige und Menschen mit Alkoholmissbrauch die billigste Art an Alkohol konsumieren, können die Ergebnisse aber als Anstoß für eine gezielte und austarierte Preisgestaltung genutzt werden. Hier liefert die Studie, auch im Hinblick auf ein erhöhtes Konsummuster in der jüngeren Generation, wichtige Erkenntnisse.

Dr. med. Thomas Stöckl

Stockwell T, et al. Does minimum pricing reduce alcohol consumption? The experience of a Canadian province. Addiction 2012; 107 (5): 912-20 